\title{
The innovative use of capillary isoelectric focusing with whole column imaging detection for carbamazepine binding to human serum albumin
}

Małgorzata Maciążek-Jurczyk*, Janusz Pawliszyn ${ }^{\#}$

Department of Chemistry, University of Waterloo, Ontario N2L 3G1, Canada

* On-leave: Department of Physical Pharmacy, School of Pharmacy with the Division of Laboratory Medicine in Sosnowiec, Medical University of Silesia, Katowice, Poland

\# Corresponding author. Tel.: 1519888 4567; fax: 15197460435

E-mail address: janusz@uwaterloo.ca (J. Pawliszyn)

\begin{abstract}
The binding of the anticonvulsant drug carbamazepine (CBZ) to human serum albumin, both without (dHSA) and in the presence of fatty acids (HSA) was studied in real time by capillary isoelectric focusing with whole column imaging detection (cIEF-WCID). Reaction mixtures at different CBZ:HSA and CBZ:dHSA molar ratios $(0: 1 \div 25: 1)$ were prepared in phosphate buffer saline (PBS) solution at a physiological $\mathrm{pH}$ (7.4), and incubated for 0 to $72 \mathrm{~h}$ at $37^{\circ} \mathrm{C}$ in a water bath. Application of the cIEF-WCID method allowed for observations on the impact of increasing CBZ:serum albumin molar ratios on isoelectric point (pI) shifts, as well as changes in peak area and absorbance, which serve as evidence of structural alterations occurring in the protein in the presence of CBZ. The obtained cIEF-WCID results indicated that the dynamic process of complex formation is not dependent on incubation time. The presented work allowed for recognition of different types of interactions, as well as for the calculation of association constants that demonstrate the stability of the complex. This study was also designed to examine the possible impact of fatty acids (FAs) on protein stability and drug delivery in blood.
\end{abstract}

\section{Keywords:}

cIEF-WCID, human serum albumin, fatty acids, carbamazepine, isoelectric point

\section{Introduction}


The degree to which a drug binds to protein is considered to be a major determinant of drug distribution. Binding plays an important role on drug dynamics, since only the free (unbound) fraction of a given drug can interact with receptors, and consequently induce therapeutic response. The most abundant blood protein, serum albumin (SA), is produced by the liver. Albumin functions primarily as a carrier protein for steroids, fatty acids, and thyroid hormones present in blood, and plays a major role in stabilizing extracellular fluid volume by contributing to the oncotic pressure (known also as colloid osmotic pressure) of plasma [1]. The primary sequence of human serum albumin (HSA) shows that the protein consists of a single polypeptide of 585 amino acids residues containing 17 pairs of disulfide bridges, one free thiol (Cys-34), and a single tryptophan (Trp-214). The protein is composed of three structurally similar, though asymmetrical domains, I, II and III, each of which is divided into two subdomains, A and B. Albumin has two main drug-binding sites, characterized as Sudlow's site I and Sudlow's site II [2], which bind drugs selectively. Site I, also known as the warfarin binding site, is formed by a pocket in subdomain IIA of HSA [1]. Warfarin is the selective probe drug for this site [3]. Site II is located in subdomain IIIA, and is known as the benzodiazepine binding site. Ibuprofen and diazepam are selective drug probes for site II [1]. It is noteworthy to mention that many different functional groups presented on protein structure are participated in drug-protein complex stabilizing processes; these are conducted through van der Waal's force and electrostatic interactions, as well as hydrogen bonds. These interactions can lead to either specific or non-specific interactions between the protein and drug [4]. As the primary ligands for protein are fatty acids (FAs), competition in binding with drugs may occur as a result of direct displacement in the binding site or conformational changes in protein structure induced by the molecules of ligands bound in different subdomains [5]. Each HSA molecule can carry up to seven fatty acid (FA) molecules. Seven common FA binding sites have been identified for medium and long-chain fatty acids (FAs) [6], as well as monosaturated and polyunsaturated FAs [7]. The location of the binding sites of the ligands, as well as the mechanisms of competition between various ligands, can yield valuable information concerning drug design [5]. FA sites 2, 4, and 5 bind FA with high affinity, while sites 1, 3, 6, and 7 exhibit low affinity for FA [8]. The relative arrangements of the three domains are largely changed when fatty acid chains bind to protein. For protein binding, competition between ligands is more important for ligands that have very high 
binding fractions, as the increase of free fraction can be doubled. While as competitors, FAs do not seem to affect the binding of drugs to a large extent; they can simultaneously occupy several binding sites in the albumin structure, making the drug-protein complex unstable. As such, an evaluation of binding parameters for the drug-albumin complex in all existing binding sites needs to include information about changes in protein affinity towards the drug in the presence of fatty acids [5].

Information on ligand-protein-binding can be provided by the use of various techniques, such as crystallography, fluorescence spectroscopy, magnetic nuclear resonance spectroscopy (NMR), circular dichroism (CD), Fourier transformation infrared spectroscopy (FTIR), ultrafiltration, UV-VIS spectrophotometry, calorimetry $[9,10]$, and recently, capillary isoelectric focusing with whole column dynamic imaging detection (cIEF-WCID) [11,12]. Capillary isoelectric focusing (cIEF), first reported by Hjerten and Zhu [13], combines highresolution for biochemical separation gel isoelectric focusing (IEF) with capillary electrophoresis (CE). The cIEF method is used for separation of amphoteric compounds according to different isoelectric points (pIs) [14], and can be used for analyses of proteins, biological complexes, and peptides. The isoelectric focusing process can be imaged by a charge-coupled device (CCD) camera equipped in the whole column with the use of UV absorbance or laser-induced fluorescence (LIF). When coupled with dynamic imaging detection, cIEF is referred to as cIEF-whole column imaging detection (cIEF-WCID). The cIEF-WICD coupling is a new, fast, automated, and simple technique that provides high resolution, speed, and high-sensitivity separation of amphoteric biomolecules [12]. The application of WCID provides direct monitoring of diffusion processes in real time. The use of dynamic imaging detection allows for potential applications of this technique in biotechnology, such as monitoring of protein diffusion in real time and estimations of molecular weights from diffusion coefficients (D), which is a second primary attribute, besides pI, for protein characterization and analysis. For highly abundant proteins, shifts in their isoelectric point (pI) can impact the function of organs that interact with them. Purtell et al. [15] examined the effects of changes in pI on renal handling of albumin molecules. The authors showed that an increase in pI caused an increase in heterologous albumin secretion, and increased nephron permeability. Indeed, there is a wide body of evidence in the literature that reports on the disease relevance of pI [16-20]. Considering these implications, pI changes can be treated as markers for structural changes in protein. 
Today, the discovery of blood-based biomarkers for diagnosis and prognosis of diseases is of extreme importance in the scientific and clinical fields. To this extent, the currently presented work was undertaken with aims to analyze the stability of protein based on its isoelectric point (pI) value by employing the separation technique cIEF-WCID. The presented results are followed by a discussion of the drug interaction capability of the human serum albumin in the absence (dHSA) and presence of fatty acids (HSA), as well as the influence of incubation time on formed complexes. While the interactions between drugs and proteins based on electrophoregram profiles and isoelectric points have been studied and published in the literature [11,21], for the first time, and to the best of this author's knowledge, the study of the drug - albumin complex is presented based on the binding isotherms and association constants obtained by the cIEF-WCID technique. Carbamazepine (CBZ), an antiepileptic drug, was used in this study as a model drug. To date, the binding of CBZ in the tertiary structure of human serum albumin has been analyzed through the use of volumetric and fluorescence techniques, while alterations in the secondary structure of SA in the presence of CBZ have been shown with the use of circular dichroism and FTIR spectroscopy [22]. In previous studies conducted by our team, in order to analyze the binding of CBZ to human serum albumin and investigate the free fraction of carbamazepine, different techniques were used and compared, such as solid phase microextraction (SPME) with liquid chromatography - ultraviolet detection (LC-UV), as well as the spectroscopic techniques spectrofluorescence (SFM) and proton nuclear magnetic resonance spectroscopy ( $\left.{ }^{1} \mathrm{HNMR}\right)$ [23]. The currently presented work is a continuation of the carbamazepine binding study based on another chromatographic technique, cIEF-WCID, demonstrating its innovative application.

\section{Experimental}

\subsection{Chemicals}

Methylcellulose (MC), Carrier ampholyte high resolution (CA, Pharmalytes 3.0-10.0), Tris(hydroxymethyl)aminomethane (TRIS, 99.8\%), Hydrochloric acid ( $\mathrm{HCl}, 37 \%$ ), carbamazepine (CBZ), albumin from human serum lyophilized powder (HSA), and albumin from human serum lyophilized, fatty acid-free (dHSA), were purchased from Sigma-Aldrich, CA. The pI markers $(4.14,6.14,8.18)$ were a gift from Dr. T. Huang (Advanced 
Electrophoresis Solutions, Ltd, CA). O-Phosphoric acid 85\% $\left(\mathrm{H}_{3} \mathrm{PO}_{4}\right)$ and methanol $\left(\mathrm{CH}_{3} \mathrm{OH}\right)$ were provided by Fisher Scientific (CA). Sodium hydroxide $(\mathrm{NaOH})$ was purchased from Caledon Laboratories LTD, CA. Water was purified with an ultrapure water system (Thermo Scientific Barnstead NanoPure system). Acrodisc 25 mm syringe filters were obtained from Pall Life Sciences, USA. $1.5 \mathrm{~mL}$ Microcentrifuge tubes were obtained from Fisher Scientific (USA). Separation was performed on cartridges with silica capillary tubes (Protein Simple) with an effective length of $50 \mathrm{~mm}, 100 \mu \mathrm{m}$ inside diameter (ID), and 200 $\mu \mathrm{m}$ outside diameter (OD). The multi-use centrifuge device Br4i was provided by the Jouan company. All the chemicals employed in this study were of analytical reagent grade and used without purification.

\subsection{Solutions and sample preparation}

A 0.01 M Tris(hydroxymethyl)aminomethane-Hydrochloric acid (TRIS-HCl) solution at pH adjusted to 7.4 as necessary to mimic physiological conditions was prepared by dissolving $0.6267 \mathrm{~g}$ of TRIS in $42.5 \mathrm{~mL}$ of $0.1 \mathrm{M} \mathrm{HCl}$, with the appropriate amount of purified water added to obtain $500 \mathrm{~mL}$ total volume. A stock solution of carbamazepine (CBZ, $1.37 \mathrm{x} \mathrm{10^{-1 }}$ $\mathrm{M})$ was prepared in methanol. Working solutions of CBZ at concentrations of $5.5 \times 10^{-6} \mathrm{M}-$ $1.37 \times 10^{-3} \mathrm{M}$ were prepared by dilution of stock solution in methanol. The final concentration of methanol was $1 \%(\mathrm{v} / \mathrm{v})$. Stock solutions of human serum albumin, both in the absence (dHSA) and presence of fatty acids (HSA) $\left(2.2 \times 10^{-4} \mathrm{M}\right)$ were prepared in TRISHCl pH 7.4. The CBZ:dHSA and CBZ:HSA molar ratios used for analyses were 0:1 - 25:1. All reaction mixtures were prepared in triplicate.

\subsection{Capillary isoelectric focusing (cIEF) - whole column imaging detection (WCID) system}

The cIEF-WCID experiments were recorded on an iCE280 analyzer (Convergent Bioscence, Toronto, Canada) and performed at $280 \mathrm{~nm}$. The used separation column was a fluorocarbon internally coated fused-silica capillary supported by a cartridge (Protein Simple, Toronto, Canada). The whole column ultraviolet (UV) absorption image was captured by a camera, 
which includes an imaging lens and a charge-coupled device (CCD) sensor. The iCE280 analyzer included quantitation software for rapid batch reprocessing of electrophoregrams.

Human serum albumin, both in the absence (dHSA) and presence of fatty acids (HSA), was dissolved into a lower molecular weight carrier ampholyte (CA) solution containing filtered methylcellulose (MC) and two small molecule external pI markers (4.14, 6.14 or 8.18). Final concentrations of CA, MC, and pI markers were approximately 5\% (v/v), 0.35\% (v/v), and $5 \%$, respectively. Sample solutions (final volume $200 \mu \mathrm{L}$ ) were prepared in a $1.5 \mathrm{~mL}$ centrifuge tube, then centrifuged for $5 \mathrm{~min}\left(3000 \mathrm{rpm}, 4^{\circ} \mathrm{C}\right.$ ).

The capillary was manually rinsed with nanopure water (filtered, degased) and conditioned with a $0.5 \%(\mathrm{w} / \mathrm{v}) \mathrm{MC}$ solution for 20 min before every experiment; optimal resolution was obtained by applying air pressure on the headspaces of the nanopure water and $\mathrm{MC}$, respectively. The used cartridge was rinsed for $3 \mathrm{~min}$ with $0.5 \%(\mathrm{w} / \mathrm{v}) \mathrm{MC}$ between runs to eliminate carryover effects and regenerate the column surface. The sample solution was hydrodynamically injected into the capillary to fill the whole separation column by applying air pressure on the headspace of the sample solution. One electrolyte tank on the column cartridge was filled with a catholyte $(0.1 \mathrm{M} \mathrm{NaOH}$ dissolved in $0.1 \%(\mathrm{w} / \mathrm{v})$ methylcellulose) and the other tank with an anolyte $\left(0.08 \mathrm{M} 85 \% \mathrm{H}_{3} \mathrm{PO}_{4}\right.$ was diluted in $0.1 \%(\mathrm{w} / \mathrm{v})$ methylcellulose).

As the column cartridge was filled with the sample solution and installed into the iCE280 analyzer, a high direct current (DC) voltage $(1500 \mathrm{~V}$ for the first $2 \mathrm{~min}$, followed by $3000 \mathrm{~V}$ for the last $6 \mathrm{~min}$ ) was applied across the electrolyte tanks on the column cartridge to start the IEF process. Focusing time took $8 \mathrm{~min}$. During focusing, the CCD camera took pictures of the whole column, displaying UV absorption images every $30 \mathrm{~s}$. Exposure time was $450 \mathrm{~ms}$, averaging a number of 4 scans per cycle. During the focusing process, the current was decreased from 10.8 to $3.92 \mu \mathrm{A}$. This procedure was repeated 3 times. After each experiment, nanopure water was introduced for a few minutes into the column for cleaning.

\subsection{Incubation of dHSA and HSA in the absence and presence of carbamazepine (CBZ)}

Samples of human serum albumin $\left(5.5 \times 10^{-5} \mathrm{M}\right)$, both in the absence (dHSA) and in the presence (HSA) of fatty acids, containing two $(4.14,6.14)$ pI markers $(1 \%, \mathrm{v} / \mathrm{v})$ were incubated at $37^{\circ} \mathrm{C}$ in a $0.01 \mathrm{M}$ TRIS- $\mathrm{HCl}$ buffer solution ( $\mathrm{pH}$ 7.4) for a maximum of 3 days. 
Aliquots were taken for analysis after $0,1,4,24,49$ and $72 \mathrm{~h}$. Reaction mixtures were also incubated and analyzed at the same time points in the presence of different concentrations of carbamazepine (CBZ). Molar ratios of the incubated CBZ:dHSA and CBZ:HSA solutions were $0: 1-25: 1,0: 1-25: 1$.

\subsection{Determination of binding isotherm and association constant}

Isotherms of carbamazepine binding to both human serum albumin samples were obtained

from the graph of the $f\left(L_{f}\right)=r$ function, where $r=\frac{L_{b}}{H S A}$, and $L_{b}$ and $L_{f}$ represent bound and free (unbound) ligand concentrations, respectively [24].

Association constants of carbamazepine to human serum albumin were evaluated with the use of the Scatchard method based on the equation [25]:

$$
\frac{r}{L_{f}}=n K_{a}-r K_{a}
$$

where $n$ represents the number of class of binding sites, and $K_{a}$ is the association constant.

\section{Results and discussion}

In the cIEF-WCID experiments, the capillary tubing contained carrier ampholytes and defatted (dHSA) and fatted (HSA) human serum albumin, in the presence and absence of carbamazepine (CBZ). The isoelectric focusing (IEF) process was initialized with the application of a high DC voltage across the electrolyte tanks on the column cartridge. Under high voltage conditions, linear $\mathrm{pH}$ gradient and focus the sample in bands or zones, along the $\mathrm{pH}$ gradient at its $\mathrm{pI}$, were established by the carrier ampholyte within a few minutes from the beginning of the experiment. At the end of the IEF process, the CCD camera (i.e., whole column imaging detector) had recorded all protein zones within the capillary column. In order to observe possible pI shifts during the incubation process, pI signals were calibrated by commercially available pI markers $(4.14,6.14$ or 8.18$)$ based on the pI calibration routine provided by the iCE280 analyzer software. 
The present paper describes the cIEF-WCID method for the analysis of defatted (dHSA) and fatted (HSA) human serum albumin interactions with carbamazepine (CBZ) at pH 7.4. Fig. 1 illustrates the typical cIEF profile of drug-free (control) defatted (Fig. 1a) and fatted (Fig. 1b) human serum albumin.

\section{Fig. 1.}

As can be seen from the images, the isoelectric spectrum of defatted human serum albumin obtained on ampholines by capillary isoelectric focusing yielded an approximate pI value of 4.9 (Fig. 1a), while in the presence of fatty acids, pI values of 4.5 and 4.9 were obtained (Fig. 1b). The observed pI variations may arise from small changes related to capillary inner wall properties during successive runs. Preliminary analysis yielded valuable information on conformational changes of HSA upon binding of fatty acids [26,27]. The observed differences in the number of isoelectric points (pIs) peaks obtained between samples indicate the probable effect of fatty acid binding on the structure of serum albumin. Moreover, due to the differentiated electrophoregrams of both dHSA and HSA, it can be concluded that fatty acids are bound mainly by the fraction of albumin with pI 4.5, and that they are absent in the albumin fraction with pI 4.9. In the seventies, Gaevskaia and Azhitskiu analyzed isoelectric fractions of healthy human serum albumin and their ability to bind bilirubin [28]. They found that bilirubin was bound mainly (80\%) by the fraction with pI 4.7, but was absent in the fraction with pI 5.5. Various deviations from this value can be found in the literature; for example, Lemma observed one distinct peak at pI 5.7, while minor fluctuations have been explained by inconsistencies in the manufacturing of the separation cartridges and differences in focusing time [21]. In order to monitor protein structural stability, human serum albumin solutions in the absence (dHSA) and presence (HSA) of fatty acids (Fig. 1) were incubated for 72 hours; recorded pI values for both dHSA and HSA samples were then used to determine protein structural stability. As large differences in the pI of proteins might have

important consequences on the structure, properties, functionality, and interaction of said proteins, pI shifts and structural alterations of SA in the presence of carbamazepine (CBZ:dHSA = CBZ:HSA 50:1) were monitored for a period of 72 hours (Fig. 2).

\section{Fig. 2.}


Fig. 2 illustrates typical cIEF separation profiles for carbamazepine-dHSA and carbamazepine-HSA complexes. Regardless of the presence of fatty acids and carbamazepine, no peak distortion or significant intensity changes were observed within the $72 \mathrm{~h}$ of the incubation period. Only insignificant variations were registered, which may arise from small changes occurring in the capillary inner wall properties during successive runs. The obtained results demonstrated the stability of the protein within a $72 \mathrm{~h}$ period of incubation. Khaldi and Shields have previously shown that the proteins observed to shift dramatically are highly glycosylated and phosphorylated [29]. In related work, Candiano et al. demonstrated that this shift in the isoelectric point is related to denaturation [30]. Lack of changes in the position of isoelectric points also established that no denaturation of protein had occurred, confirming the structural stability of the protein based on the pI value. Similarly, no significant changes were observed for the first $6 \mathrm{~h}$ on work conducted by Lemma on the influence of incubation time on the serum albumin - oxaliplatin complex. As the incubation time progressed from 6 to $12 \mathrm{~h}$, and further to $72 \mathrm{~h}$, oxaliplatin adduct-protein formation was manifested by changes in peak intensity and width, as well as shifts in the isoelectric point [21].

To investigate the carbamazepine (CBZ) - human serum albumin (dHSA, HSA) interaction as well as the influence of fatty acids on the binding affinity of serum albumin towards CBZ, cIEF analyses of the CBZ-dHSA (Fig. 3a) and CBZ-HSA (Fig. 3b) complexes were conducted.

Fig. 3.

Fig. 3 illustrates the standard cIEF profile of defatted (Fig. 3a) and fatted (Fig. 3b) human serum albumin in the absence (CBZ:dHSA $=$ CBZ:HSA $=0: 1$ ) and presence of CBZ $(\mathrm{CBZ}: \mathrm{dHSA}=\mathrm{CBZ}: \mathrm{HSA}=0.1: 1-25: 1)$. As the CBZ concentrations increased for CBZ:dHSA and CBZ:HSA from 0:1 to 1:1, the pI values and peak areas were observed to change. The spectral profiles of Fig. 3 show that the protein was significantly affected by the complexation with carbamazepine. Based on data collected from CBZ-dHSA and CBZ-HSA electrophoregrams, a relationship between $\mathrm{pI}$ and the CBZ:serum albumin molar ratio was 
found to be present, as can be seen in Fig. 4. To further illustrate this relationship, the dependence correlation between peak area and drug:albumin molar ratio is shown in Fig. 5.

\section{Fig. 4.}

The study of pI is the first attribute that needs to be established for protein characterization and analysis. As evidenced, as the molar ratio of CBZ:dHSA (Fig. 4a) and CBZ:HSA (Fig. 4b) increased from $0: 1$ to $1: 1$, the pI was noted to similarly increase. The observed shifts in the isoelectric point evidence protein structural alterations induced by carbamazepine upon protein incubation, both in the absence (dHSA) and presence (HSA) of fatty acids. For both CBZ:dHSA and CBZ:HSA, molar ratios above 1:1 produced no significant changes, indicating that saturation of the carbamazepine complex with serum albumin occurred after this point; as such, the optimum concentration of CBZ could be calculated based on these results. In addition, the obtained results allow for the conclusion that changes in isoelectric point are accompanied by modifications other than in the protein structure environment of the binding site.

In order to confirm saturation of the complex irrespective of fatty acid presence, changes in peak area as a function of carbamazepine:serum albumin molar ratio were investigated, with results presented on Fig. 5.

\section{Fig. 5.}

Fig. 5 shows that the peak area gradually changed as carbamazepine concentration increased. The cIEF results indicate that as the CBZ:dHSA molar ratio gradually increased from 0:1 to 1:1 (Fig. 5a), the peak area of the pI spectrum increased accordingly, reaching a plateau above the CBZ:dHSA 1:1 mark. This increase in the peak areas of the obtained cIEF profiles suggests the formation of the carbamazepine complex with serum albumin, which occurs as CBZ occupies readily available binding sites in the SA macromolecule. The observed plateau that occurred above the 1:1 molar ratio can be explained by the formation of drug-defatted albumin complexes and the subsequent saturation of all binding sites. As the CBZ:HSA molar ratio increased to $1: 1$, similarly to the observed findings for CBZ-defatted serum albumin system, the peak areas (pI 4.9) were observed to increase accordingly, with a noted 
saturation of all binding sites occurring as the ratio of CBZ:HSA increased from 1:1 to 25:1 (Fig. 5b).

In view of the obtained results, it can then be concluded that CBZ saturates all the binding sites of SA regardless of the presence of fatty acids, as can be observed by examination of the obtained curves representing both defatted complexes (Fig. 6a) and the fatty acid-containing SA-CBZ complex (Fig. 6b’, 6b’’).

\section{Fig. 6.}

Binding isotherms determine the character of the drug - serum albumin binding (specificity of binding sites in a particular class of binding). A straight line indicates a non-specific ligand binding with the hydrophobic surface of albumin. The exponential saturation curve reaching a plateau characterizes specific binding sites with high- and low-affinity binding capacities. However, a simultaneously specific and non-specific model of interaction is illustrated by the curve with course between the straight and the plateau [24]. The shape of the isotherms indicates the specific nature of the interaction of CBZ with serum albumin, both in the absence (Fig. 6a) and presence of fatty acids (Fig. 6b',6b”). No changes in binding isotherms above the free concentration of carbamazepine $5.08 \times 10^{-4} \mathrm{M}$ have been observed. This is in accordance with the results obtained in an analysis of peak areas and pI shifts, confirming the saturation of the complex at the molar ratio of CBZ:dHSA = CBZ:HSA 1:1. The association constant $\mathrm{K}_{\mathrm{a}}$ of the carbamazepine-albumin complex, as well as the number of present different classes of binding sites $n$ were determined using the Scatchard equation (1). Association constants were then calculated using non-linear regression based on the Levenberg - Marquardt algorithm (Table 1).

\section{Table 1}

A determination of association constants and number of classes of binding sites based on Equation (1) point to one class of high affinity binding sites between carbamazepine and serum albumin without fatty acids (dHSA), and two classes of high and low affinity binding sites via hydrophobic interaction $(n=0.9)$. The obtained association constants are $6.94 \times 10^{4}$ $\mathrm{M}^{-1}$ for one fraction of dHSA ( $\mathrm{pI}=4.9$ ), and $1.41 \times 10^{4} \mathrm{M}^{-1}$ and $0.40 \times 10^{4} \mathrm{M}^{-1}$ for two 
fractions of HSA, at pI $=4.9$ and 4.5, respectively. These results are in agreement with carbamazepine-serum albumin studies recently conducted by Bojko et al., who have through application of the SPME method demonstrated the existence of two high and low affinity binding sites for CBZ on the human albumin macromolecule (HSA), using analysis of Lineweaver-Burk plots [23]. The obtained binding location results also further confirm findings obtained by Kim et al., who have reported the existence of an additional binding site based on chromatographic analysis [31]. Additionally, the obtained values for the association constants presented in Table 1 share a marked similarity to data presented by Bojko et al. based on SPME methodology for parameters set to $\mathrm{MeOH} 1 \%(\mathrm{v} / \mathrm{v})\left(\mathrm{K}_{\mathrm{aI}}=3.38 \times 10^{4} \mathrm{M}^{-1}\right.$ and $\mathrm{K}_{\mathrm{aII}}=0.75 \times 10^{4} \mathrm{M}^{-1}$ ). In this series of studies, the influence of methanol on protein affinity towards CBZ was investigated, with findings revealing that a methanol content above $1 \%$ $(\mathrm{v} / \mathrm{v})$ changed the interaction in the CBZ-HSA complex. The similarity between the figures obtained by cIEF association constants in the currently presented work and those presented for $\mathrm{MeOH} 1 \%(\mathrm{v} / \mathrm{v})$ confirm this phenomenon. However, use of the fluorescence technique, which provides information regarding structural changes in protein structure both for the Scatchard and Lineweaver-Burk methods, revealed the existence of only one high affinity binding site class for CBZ, located in the tertiary structure of the albumin macromolecule, with an association constant of $\mathrm{K}_{\mathrm{a}} 2.5 \times 10^{4} \mathrm{M}^{-1}$ [23]. To evaluate the binding parameters using the fluorescence technique, Bojko et al. had to adjust the CBZ:protein molar ratios so as to obtain maximum quenching of serum albumin fluorescence by the ligand, while saturation of the complex CBZ with albumin was achieved at a molar ratio of CBZ:SA 20:1. The necessity of maximum fluorescence quenching, opposite to the principles applied for the cIEF method, can be pointed as a possible reason for the discrepancy in the number of classes of binding sites. MacKichan et al [32], similarly to Bojko et al., compared plasma protein binding (PPB\%) with literature data obtained with different techniques; in this investigation, the authors observed significant discrepancies that correlated to differences in drug:protein molar ratios used for different experiments. Notably, Bojko et al. highlighted that reported discrepancies can arise from fundamentally different principles of methods. Inspite of that, our results found that the one class of binding sites in the CBZ-dHSA complex, as well as the value of the association constant correlate greatly to the high affinity binding site values calculated by Bojko et al. The binding of carbamazepine (CBZ) to human serum albumin (HSA) was investigated under simulated physiological conditions by Kalanur et al. with the 
use of spectroscopic and volumetric techniques [22]. In this study, the formation of the CBZHSA complex in the IIA subdomain via the static quenching effect of CBZ was monitored, yielding a $2.081+/-0.023 \times 10^{4} \mathrm{M}^{-1}$ association constant. Fluorimetric and voltammetric results further revealed that the hydrophobic interaction was a predominant intermolecular force for stabilizing the complex. The data obtained using the presented cIEF technique is attributed to absorbance at $280 \mathrm{~nm}$; use of this technique, similarly to the use of the spectrofluorescence technique, presents the possibility of future analyses of protein regions containing tryptophanyl and tyrosil aminoacids residues, which absorb radiation at the 280 nm wavelength. One tryptophanyl Trp-214 residue is located in subdomain IIA, Sudlow's site I [1] while tyrosil residues are located in subdomains IIA (Tyr-263) and IIIA (Tyr-401, 411, 497), Sudlow's site II [33]. These hydrophobic pockets, located on subdomains IIA and IIIA, are the main drug-binding sites most commonly found with the use of fluorescence technique in SA. The recorded cIEF data allows us to identify subdomains IIA and IIIA as specific high- and low-affinity binding sites, respectively, which is in accordance with recent reports $[22,23]$. The ability of cIEF to register both classes of binding sites makes the technique more practical than spectrofluorescence, as cIEF forgoes the necessity of inner filter correction. Inner filter effects include reabsorption when molecules absorbs at the wavelengths at which the fluorophore emits radiation; consequently, some or all of the photons emitted by the fluorophore may be absorbed again. In addition, the inner filter effect can cause inconsistences in excitation light emission throughout the solution, where only a small percentage of the excitation light reaches the fluorophores that are visible for the detection system. Inner filter effects change the spectrum and intensity of the emitted light, and as such, they must be considered when analyzing the emission spectrum of fluorescent light [34,35]. In this context, it is worth noting that the intensity of observed fluorescence has to be corrected for the inner filter when the absorbance of the ligand is between 0.05 and 0.3 [35,36]. Based on previously reported data, the absorbance of carbamazepine at $280 \mathrm{~nm}$ reaches almost 0.7 A.U [37]. As little to no data is available concerning appropriate corrections for inner filter effects for this particular compound, the currently presented data cannot be reliably compared with past spectrofluorescence results. In addition, the lack of current corrections for data obtained in the corresponding CBZ spectrofluorescence study also further explains why the obtained CIEF data is significantly better correlated to data obtained with application of the SPME method [23]. 
A comparison of association constants calculated for CBZ-HSA and CBZ-dHSA complexes has shown that the presence of fatty acids causes an alteration within a hydrophobic region of the HSA structure, resulting in a weaker interaction between ligand and fatty isoforms of the protein (Table 1). As subdomains IIA and IIIA are locations for primary fatty acid binding sites, the competition between fatty acids and carbamazepine can then be assumed to occur. Bojko et al. also compared low affinity binding sites based on NMR and SPME techniques [23]. NMR studies provide information regarding the functional group of a given drug involved in complex formations. As no changes in chemical shifts were observed as a result of very low concentrations of the ligand, a proper comparison of CIEF and SPME methods cannot be made at this point in time, although application of the cIEF technique for simultaneous analysis of high- and low-affinity binding sites seems to be highly reasonable.

\section{Conclusion}

The presented data provides useful information concerning drug-albumin interactions, and clearly illustrates the quality of information obtained by the use of cIEF-WCID towards analysis of carbamazepine binding to human serum albumin, both in the absence and presence of fatty acids, and monitored based on changes in pI values, peak areas, and association constants. Based on the presented data, it can be hypothesized that the binding of carbamazepine has a significant effect on the structural stability of the protein. In the present report, results obtained with cIEF-WCID were compared with methods having fundamentally different principles, such as protein fluorescence quenching, proton nuclear magnetic resonance, and solid phase microextraction (SPME), confirming that the collection of complete information regarding local drug-amino acids interactions as well as the ability of the protein to make complex formations is attainable with the use of cIEF-WCID. Although cIEF-WCID cannot be treated as a competitive technique, as it does not provide information obtained by methods such as SPME on free drug concentrations (an important factor in drug development studies), the determination of association constants distinguishes this basic method among other separation methodology. It is noteworthy that pI changes can be treated as markers for structural changes in protein. Moreover, this technique provides immediate high resolution results, an application of clinical interest that might be useful for rapid diagnoses of diseases. 


\section{Acknowledgement}

The authors thank Dr. Tiemin Huang (Advanced Electrophoresis Solutions AES, Ltd., Cambridge, Ontario, Canada N3H 4W5) for donation of pI markers, and Dr. Jiaqi Wu, Principal Scientist in Protein Simple, for donation of cartridges.

\section{References}

[1] U. Kragh-Hansen, V.T. Chuang, M. Otagiri, Practical aspects of the ligand binding and enzymatic properties of human serum albumin, Biol. Pharm. Bull. 25 (2002) 695-704.

[2] G. Sudlow, D.J. Birkett, D.N. Wade, The characterization of two specific drug binding sites on human serum albumin, Mol. Pharmacol. 11 (1975) 824-832.

[3] I. Petitpas, A.A. Bhattacharya, S. Twine, M. East, S. Curry, Crystal structure analysis of warfarin binding to human serum albumin: anatomy of drug site I. J. Biol. Chem. 276 (2001) 22804-22809.

[4] K. Jozwiak, W.J. Lough, I.W. Wainer, Drug Stereochemistry: Analytical Methods and Pharmacology, Third Edition, Informa Healthcare, 2012.

[5] B. Bojko, A. Sułkowska, M. Maciążek-Jurczyk, J. Równicka, W.W. Sułkowski, The influence of dietary habits and pathological conditions on the binding of theophylline to serum albumin, J. Pharm. Biomed. Anal. 52 (2010) 384-390.

[6] A.A. Bhattacharya, T. Grune, S. Curry, Crystallographic analysis reveals common modes of binding of medium and long-chain fatty acids to human serum albumin, J. Mol. Biol. 303 (2000) 721-732.

[7] I. Petitpas, T. Grune, A.A. Bhattacharya, S. Curry, Crystal structures of human serum albumin complexed with monounsaturated and polyunsaturated fatty acids, J. Mol. Biol. 314 (2001) 955-960.

[8] J.R. Simard, P.A. Zunszain, J.A. Hamilton, S. Curry, Location of high and low affinity fatty acid binding sites on human serum albumin revealed by NMR drug-competition analysis, J. Mol. Biol. 361 (2006) 336-351.

[9] H. Gao, L. Lei, J. Liu, Q. Kong, X. Chen, Z. Hu, The study on the interaction between human serum albumin and a new reagent with antitumour activity by spectrophotometric methods, J. Photochem. Photobiol. 167 (2004) 213-221. 
[10] C. Bertucci, G. Ascoli, G. Uccello-Barretta, L. Di Bari, P. Salvadori, The binding of 5fluorouracil to native and modified human serum albumin: UV, CD, and 1H and 19F NMR investigation, J. Pharm. Biomed. Anal. 13 (1995) 1087-1093.

[11] T. Lemma, R. Mandal, X.F. Li, J. Pawliszyn, Investigation of interaction between human hemoglobin A0 and platinum anticancer drugs by capillary isoelectric focusing with whole column imaging detection, J. Sep. Sci. 31 (2008) 1803-1809.

[12] Z. Liu, T. Lemma, J. Pawliszyn, Capillary isoelectric focusing coupled with dynamic imaging detection: A one-dimensional separation for two-dimensional protein characterization, J. Proteome Res. 5 (2006) 1246-1251.

[13] S. Hjerten, M. Zhu, Adaptation of the equipment for high-performance Electrophoresis to Isoelectric Focusing. J. Chromatogr. A 346 (1985) 265-270.

[14] F. Kilar, Recent applications of capillary isoelectric focusing. Electrophoresis 24 (2003) 3908-3916.

[15] J.N. Purtell, A.J. Pesce, D.H. Clyne, W.C. Miller, V.E. Pollak, Isoelectric point of albumin: effect on renal handling of albumin. Kidney Int. 16 (1979) 366-76.

[16] M.C. Plucinsky, J.J. Prorok, J.A. Alhadeff, Beta-Hexosaminidase from colon and sera of dukes-classified colorectal cancer patients: activity levels, isozyme patterns, and kinetic properties, J. Natl. Cancer Inst. 77 (1986) 57-62.

[17] I.L. MacGregor, D. Zakim, A cause of hyperamylasemia associated with chronic liver disease, Gastroenterology 72 (1977) 519-523.

[18] T. Saitoh, J.P. Changeux, Change in state of phosphorylation of acetylcholine receptor during maturation of the electromotor synapse in Torpedo marmorata electric organ, Proc. Natl. Acad. Sci. USA 78 (1981) 4430-4434.

[19] J.F. Coy, D. Dressler, J. Wilde, P. Schubert, Mutations in the transketolase-like gene TKTL1: clinical implications for neurodegenerative diseases, diabetes and cancer, Clin. Lab. 51 (2005) 257-273.

[20] O. Alzate, C. Osorio, R.M. DeKroon, A. Corcimaru, H.P. Gunawardena, Differentially charged isoforms of apolipoprotein $\mathrm{E}$ from human blood are potential biomarkers of Alzheimer’s disease, Alzheimer's Research \& Therapy 6 (2014) 43 -54.

[21] T. Lemma, J. Pawliszyn, Human serum albumin interaction with oxaliplatin studied by capillary isoelectric focusing with the whole column imaging detection and spectroscopic method. J. Pharm. Biomed. Anal. 50 (2009) 570-575. 
[22] S.S. Kalanur, J. Seetharamappa, V.K. Kalalbandi, Characterization of interaction and the effect of carbamazepine on the structure of human serum albumin, J. Pharm. Biomed. Anal. 53 (2010) 660-666.

[23] B. Bojko, D. Vuckovic, J. Pawliszyn, Comparison of solid phase microextraction versus spectroscopic techniques for binding studies of carbamazepine, J. Pharm. Biomed. Anal. 66 (2012) 91-99.

[24] Z. Taira, H. Terada, Specific and non-specific ligand binding to serum albumin, Biochem. Pharmacol. 34 (1985) 1999-2005.

[25] T. Hiratsuka, Conformational changes in the 23-kilodalton NH2-terminal peptide segment of myosin ATPase associated with ATP hydrolysis, J. Biol. Chem. 265 (1990) 18786-18790.

[26] S. Fujiwara, T. Amisaki, Molecular dynamics study of conformational changes in human serum albumin by binding of fatty acids, Proteins. 64 (2006) 730-739.

[27] S. Fujiwara, T. Amisaki, Identification of High Affinity Fatty Acid Binding Sites on Human Serum Albumin by MM-PBSA Method, Biophys. J. 94 (2008) 95-103.

[28] V.A. Gaevskaia, GIu. Azhitskiū, Isoelectric fractions of healthy human serum albumin and their ability to bind bilirubin, Ukr. Biokhim. Zh. 50 (1978) 735-738.

[29] N. Khaldi, D. Shields, Shift in the isoelectric-point of milk proteins as a consequence of adaptive divergence between the milks of mammalian species, Biology Direct. 6 (2011) 4048.

[30] G. Candiano, G.M. Ghiggeri, G. Delfino, C. Queirolo, G. Vecchio, Isoelectric focusing in low-denaturing media: Visualization in renal disease of variation of the isoelectric point of albumin not related to a remarkable conformational variation, J. Chromatogr. 374 (1986) 279-288.

[31] H.S. Kim, D.D Hage, Chromatographic analysis of carbamazepine binding to human serum albumin, J. Chromatogr. B: Analyt. Technol. Biomed. Life Sci. 816 (2005) 57-66.

[32] J.J. MacKichan, E.M. Zola, Determinants of carbamazepine and carbamazepine 10,11epoxide binding to serum protein, albumin and alpha 1-acid glycoprotein, Br. J. Clin. Pharmacol. 18 (1984) 487-493.

[33] J. Kang, Y. Liu, M.X. Xie, S. Li, M. Jiang, Y.D. Wang, Interactions of human serum albumin with chlorogenic acid and ferulic acid, Biochim. Biophys. Acta. 1674 (2004) 205214. 
[34] A. Sharma, S.G. Schulman, Introduction to Fluorescence Spectroscopy. Wiley Interscience, 1999.

[35] J.R. Lakowicz, Principles of Fluorescence Spectroscopy. Kluwer Academic/Plenum Publishers, 1999.

[36] E.P. Kirby, in: R.F. Steiner, I. Weinryb (Ed.), Fluorescence Instrumentation and Methodology, Inc., Maryland, 1970, 39-42.

[37] R. Thilak Kumar, S. Umamaheswari, FTIR, FTR and UV-Vis Analysis of Carbamazepine, RJPBCS 2 (2011) 685-693. 


\section{Table 1}

Table 1 Association constants $\left(K_{a}\right)$ and number of binding sites (n) for one class of drug binding sites in albumin molecule determined for carbamazepine-human serum albumin complex, in the absence (CBZ-dHSA) and presence of fatty acids (CBZ-HSA).

\begin{tabular}{ccc}
\hline & \multicolumn{3}{c}{$\mathbf{K}_{\mathbf{a}}\left[\mathbf{M}^{-1}\right], \mathbf{n}$} \\
\hline CB 4.9 & pI 4.5 \\
\hline CBZ-dHSA & $6.94 \times 10^{4}, 0.9$ & - \\
\hline
\end{tabular}


a)

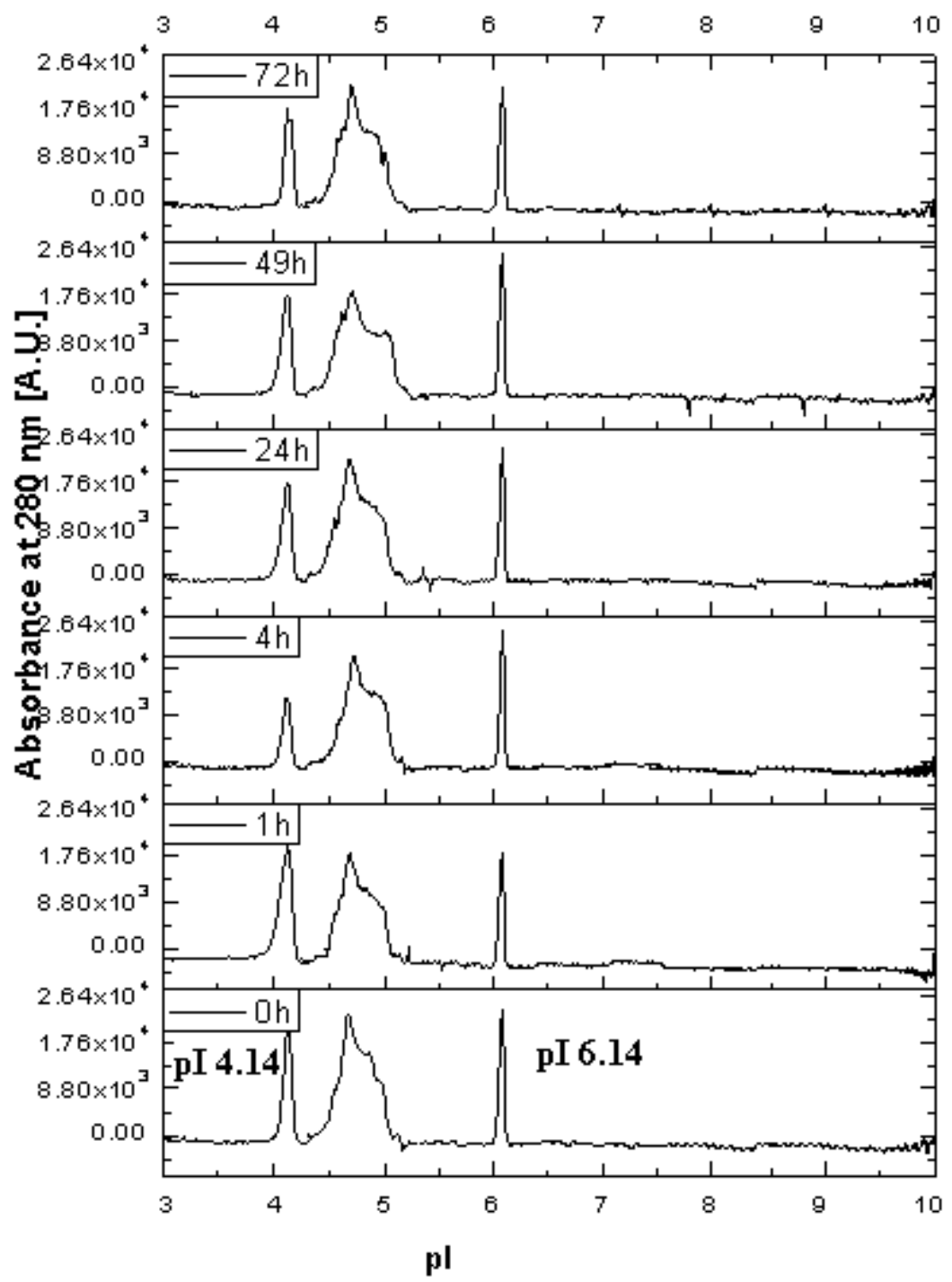




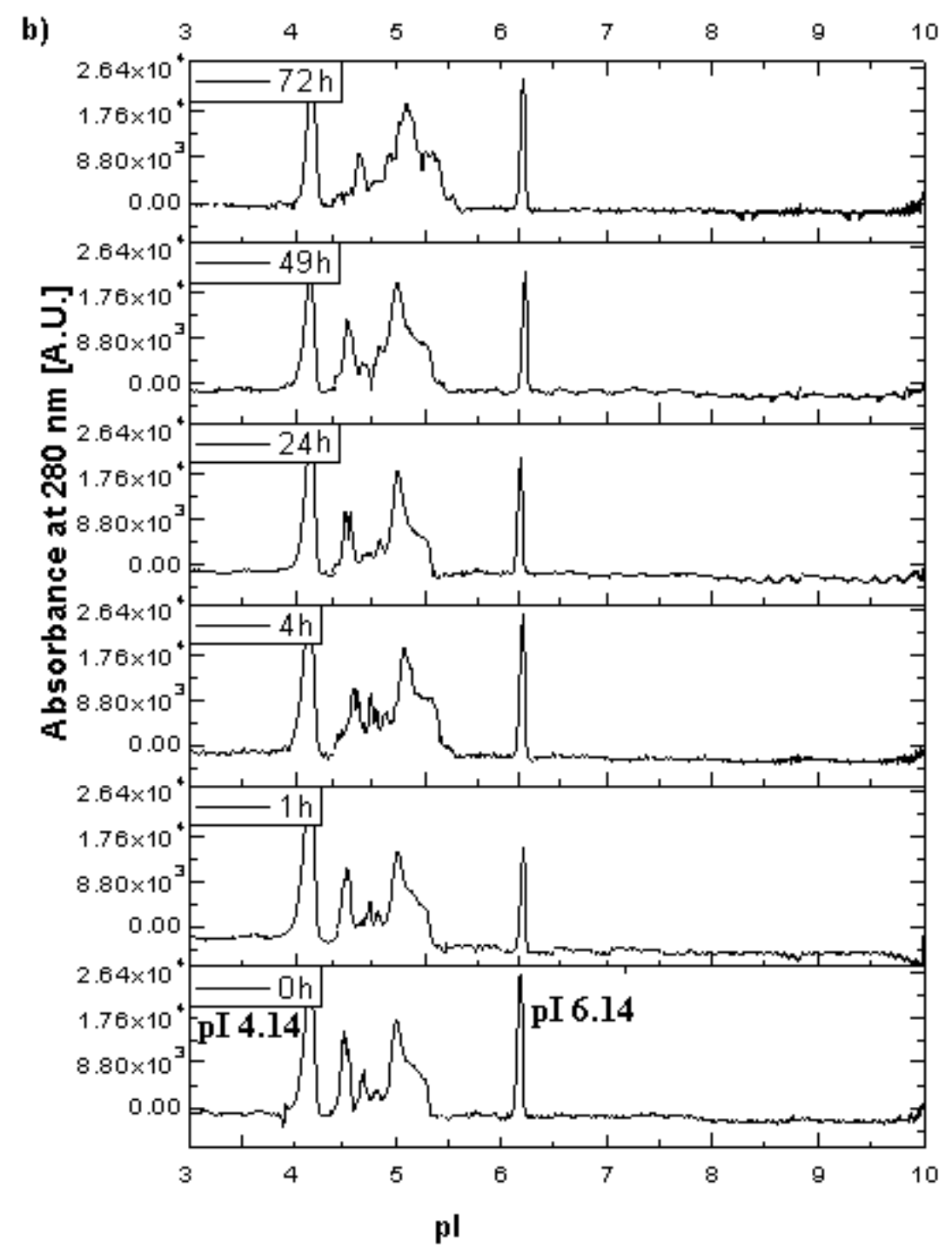

Fig. 1. cIEF analysis of a) dHSA and b) HSA at concentration $5.5 \times 10^{-5} \mathrm{M}$. Samples were incubated for $72 \mathrm{~h}$ at $37^{\circ} \mathrm{C}$. 
a)

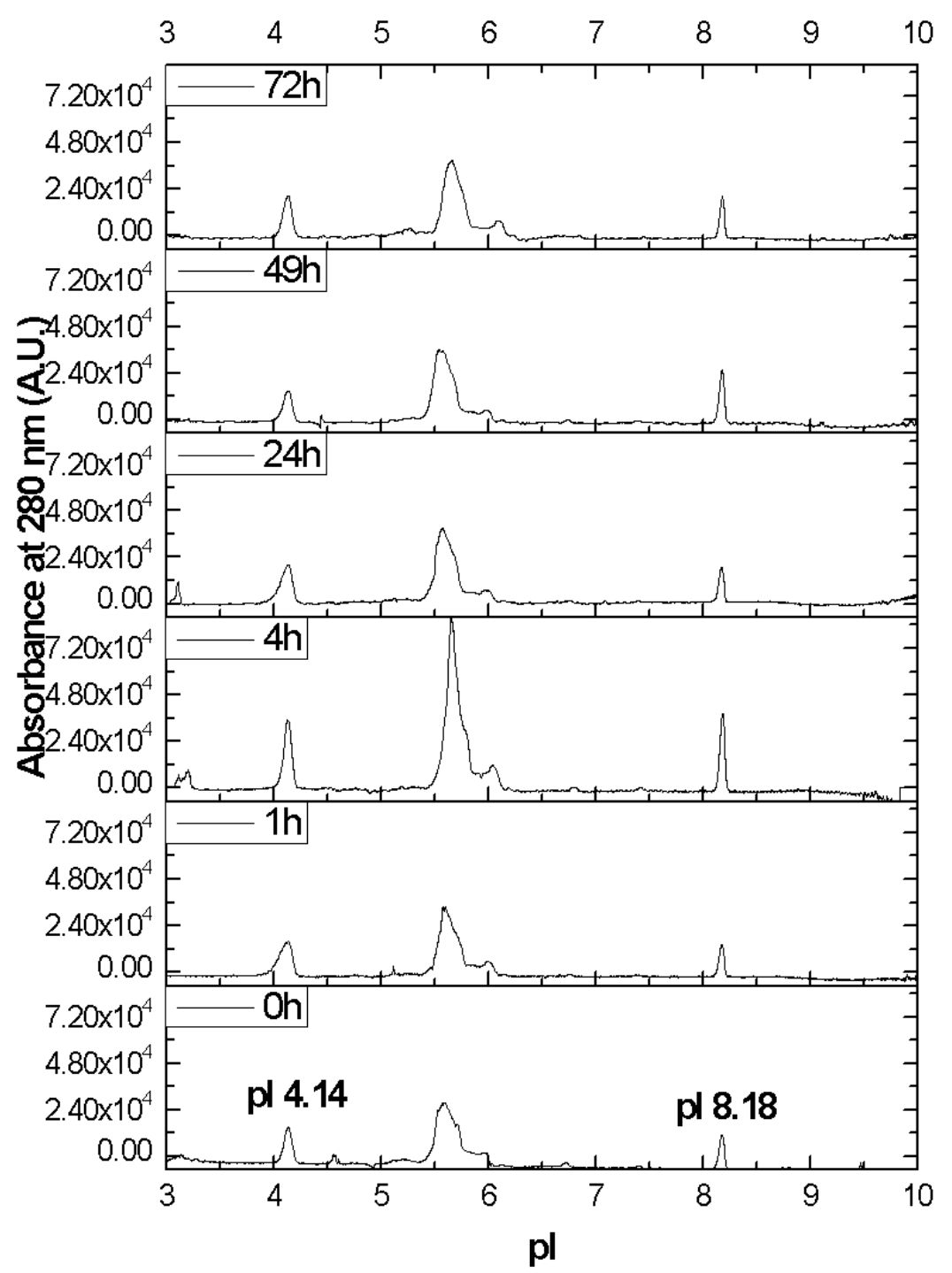


b)

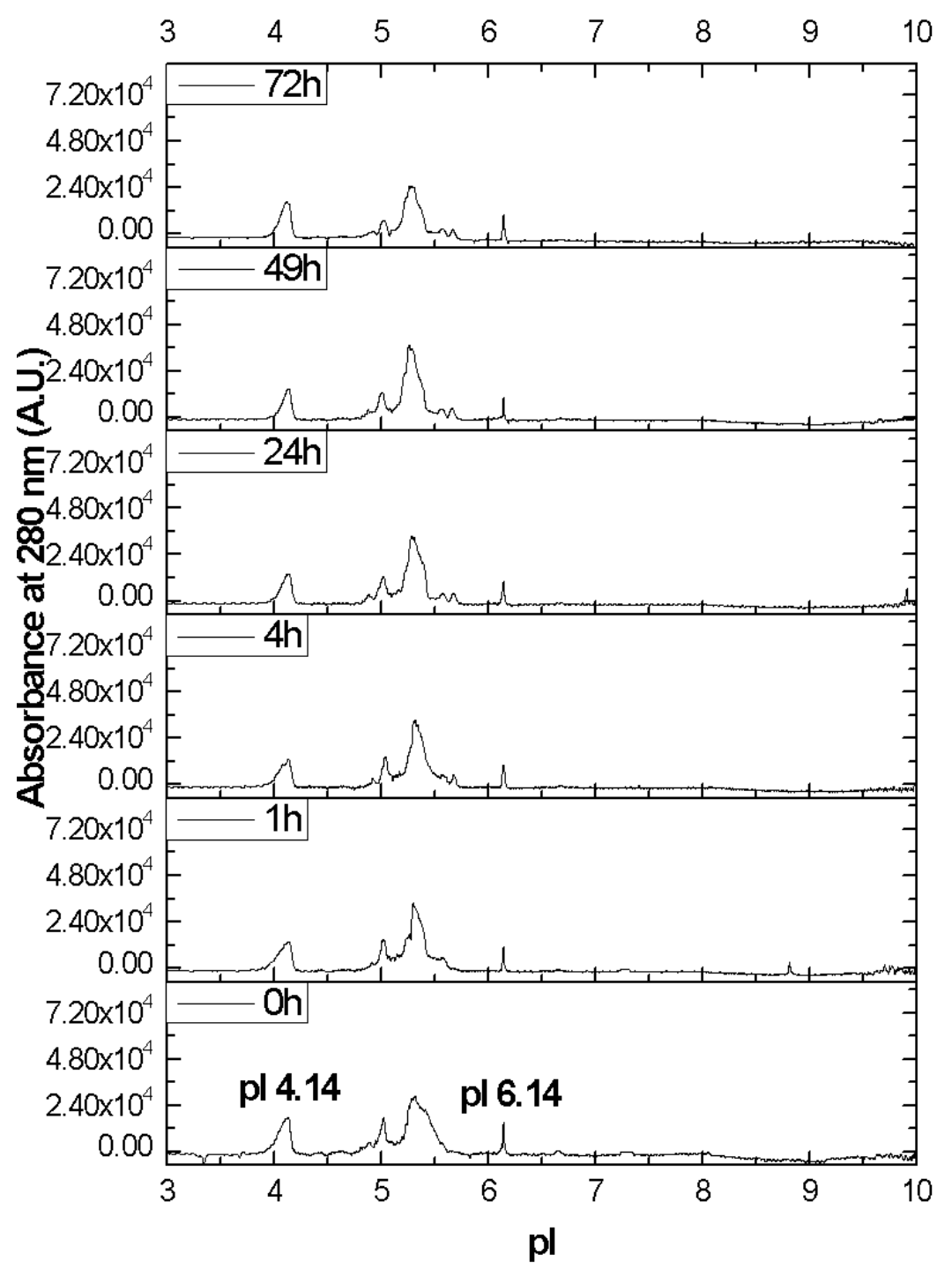

Fig. 2. cIEF analysis of a) dHSA and b) HSA in the presence of carbamazepine (CBZ:dHSA $=$ CBZ:HSA 50:1). Samples were incubated for $72 \mathrm{~h}$ at $37^{\circ} \mathrm{C}$. 


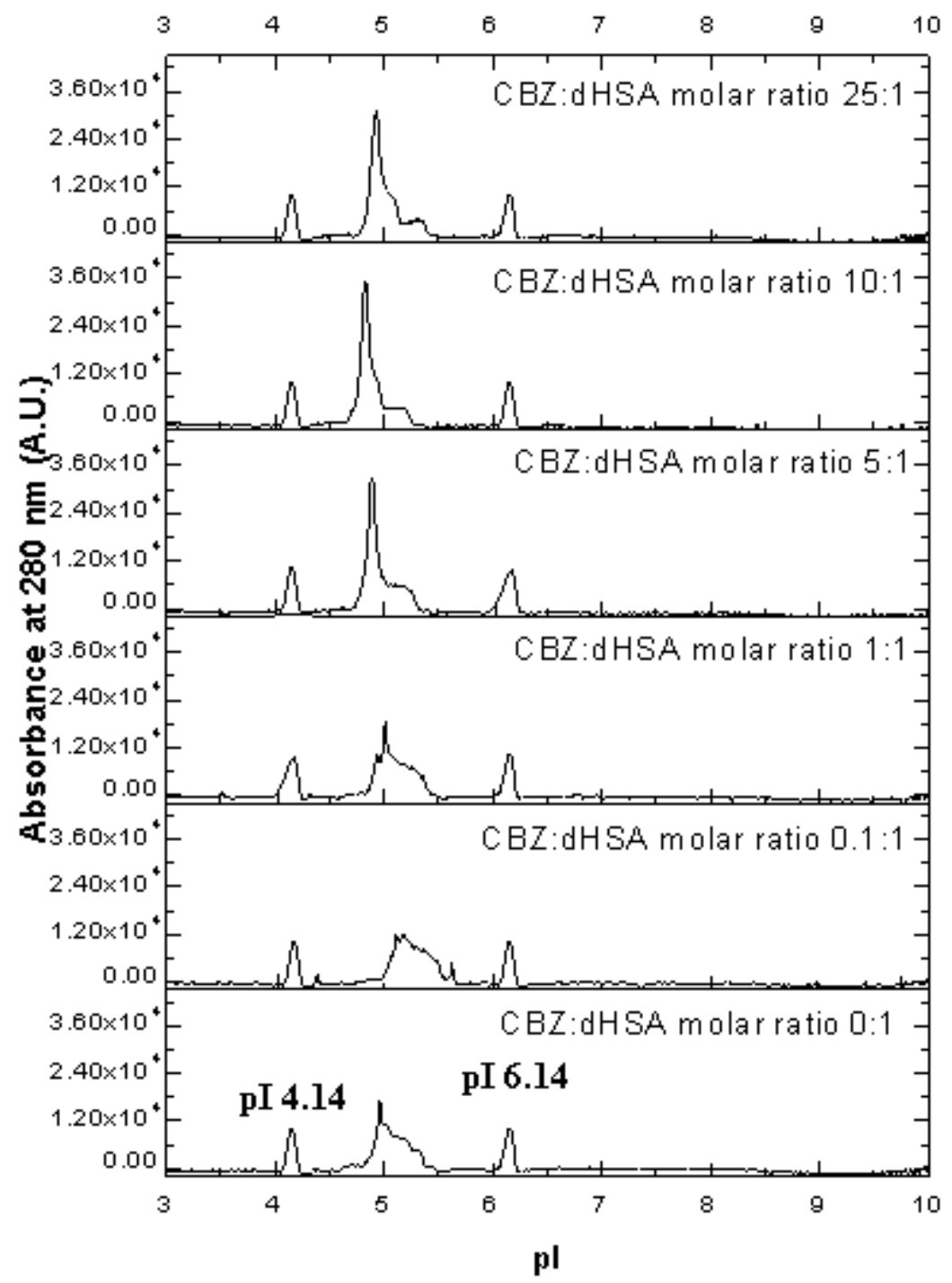


b)

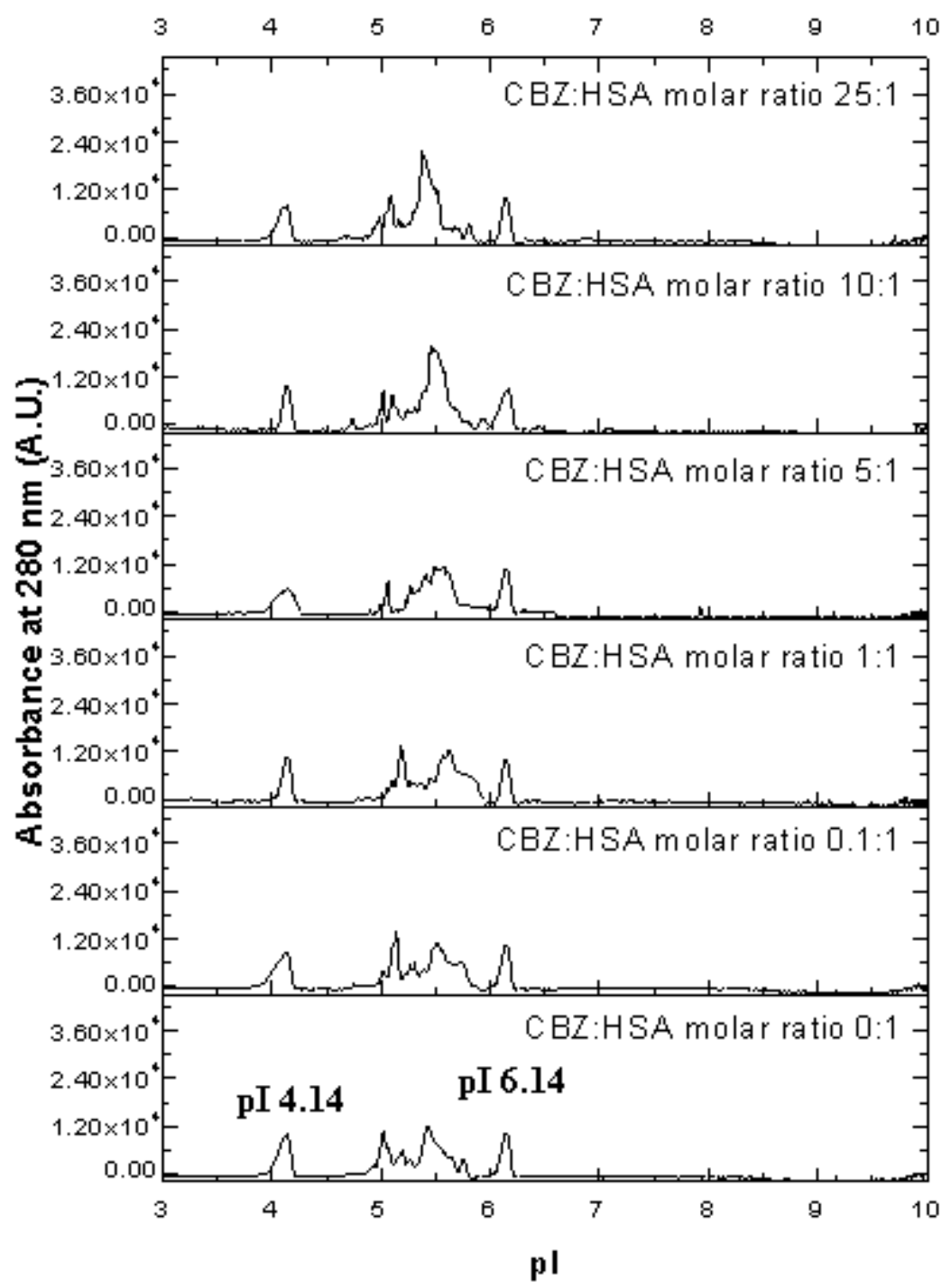

Fig. 3. Electrophoregram of a) dHSA and b) HSA in the absence (CBZ:dHSA 0:1, CBZ:HSA 0:1) and presence of CBZ (CBZ:dHSA 0.1:1 - 25:1, CBZ:HSA 0.1:1 - 25:1). 
a)

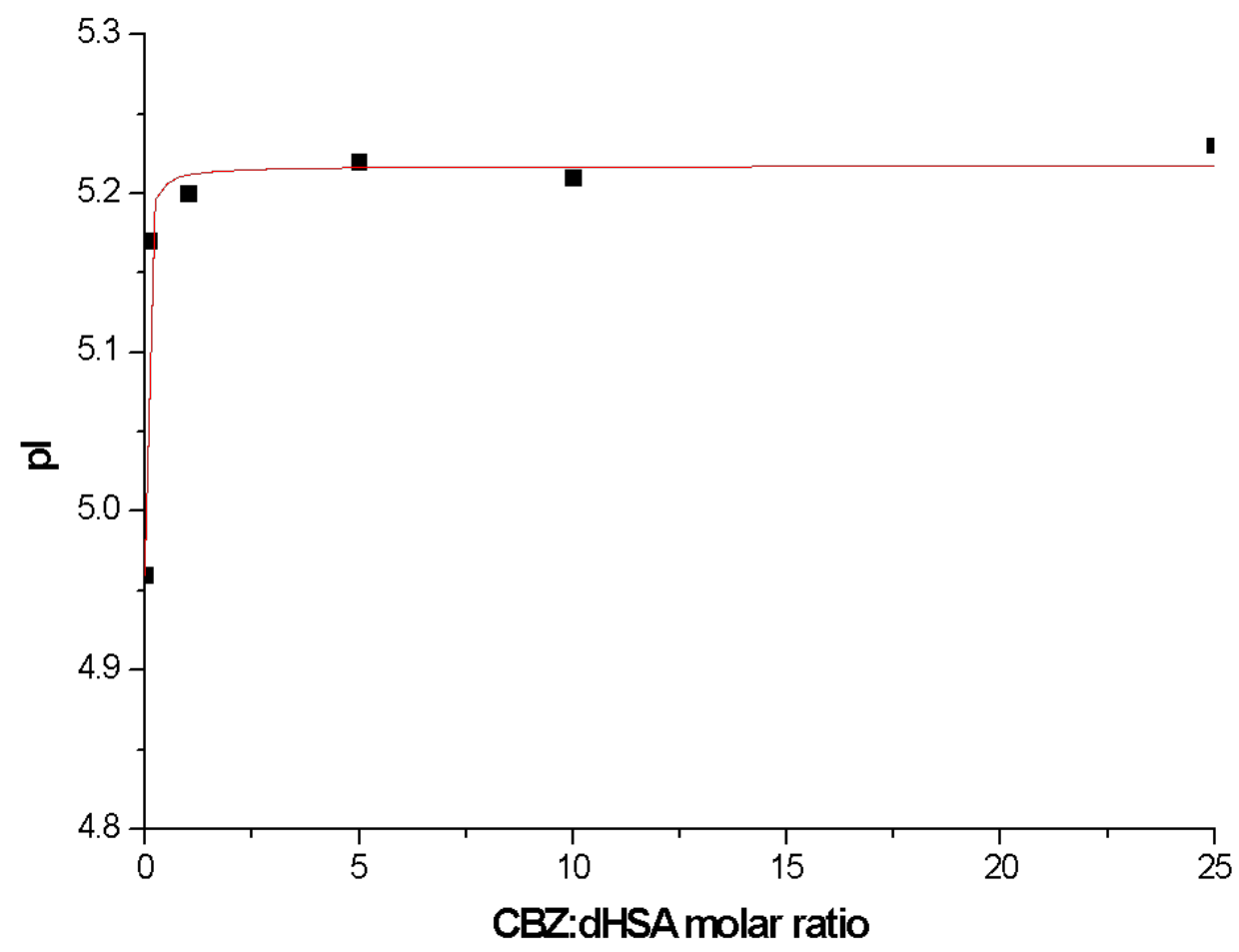


b)

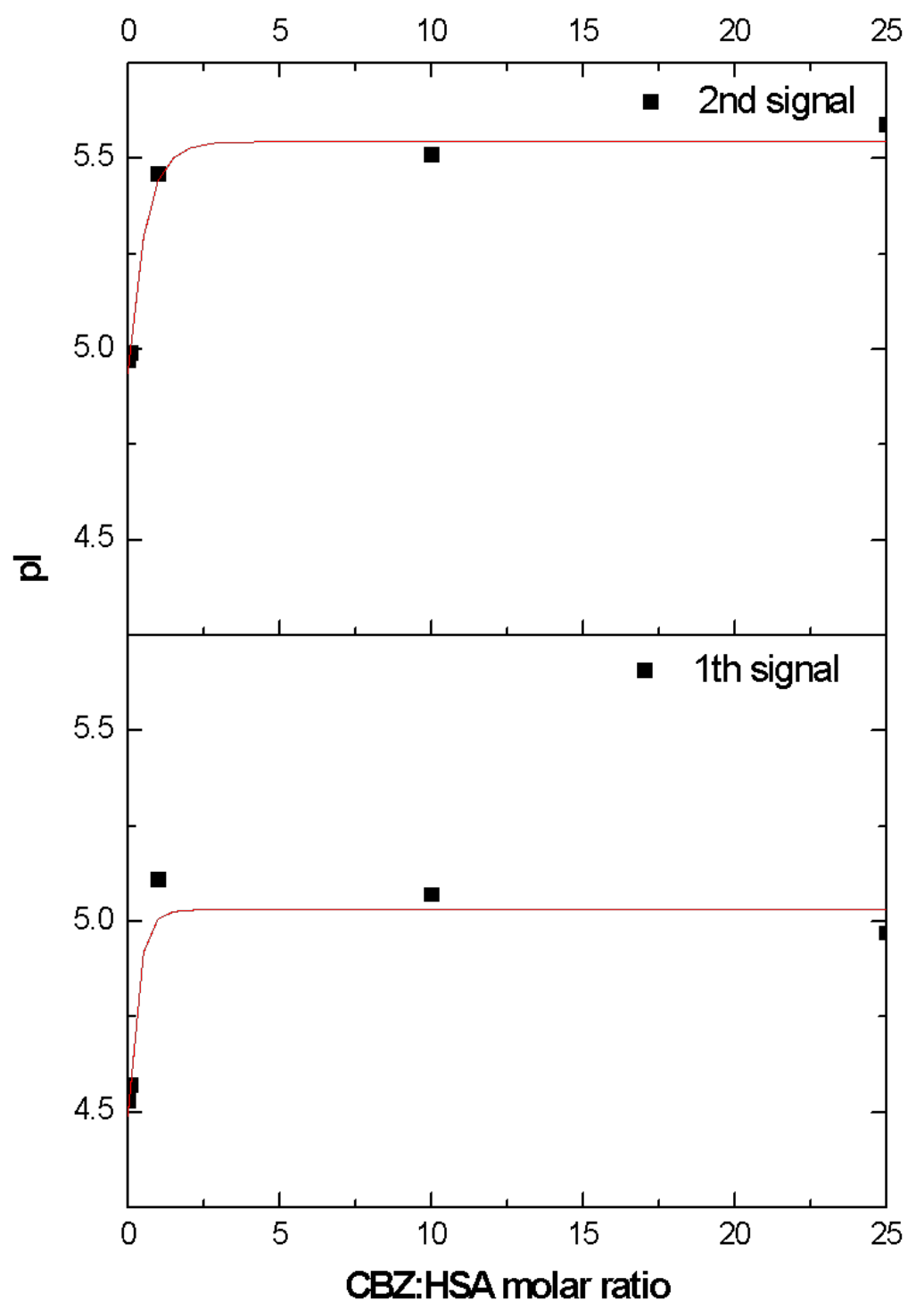

Fig. 4. Dependence between pI and a) CBZ:dHSA and b) CBZ:HSA molar ratios. 
a)

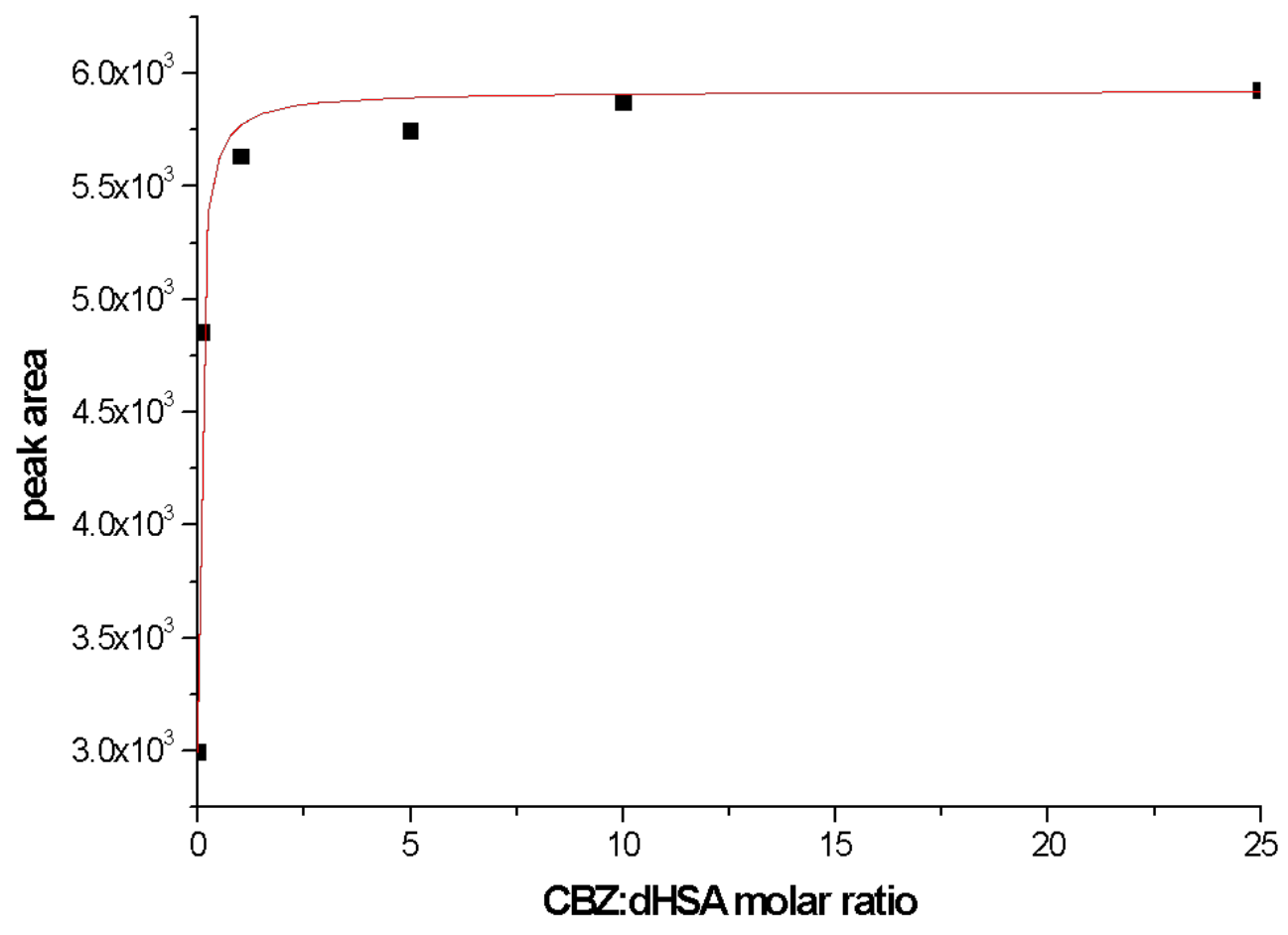


b)

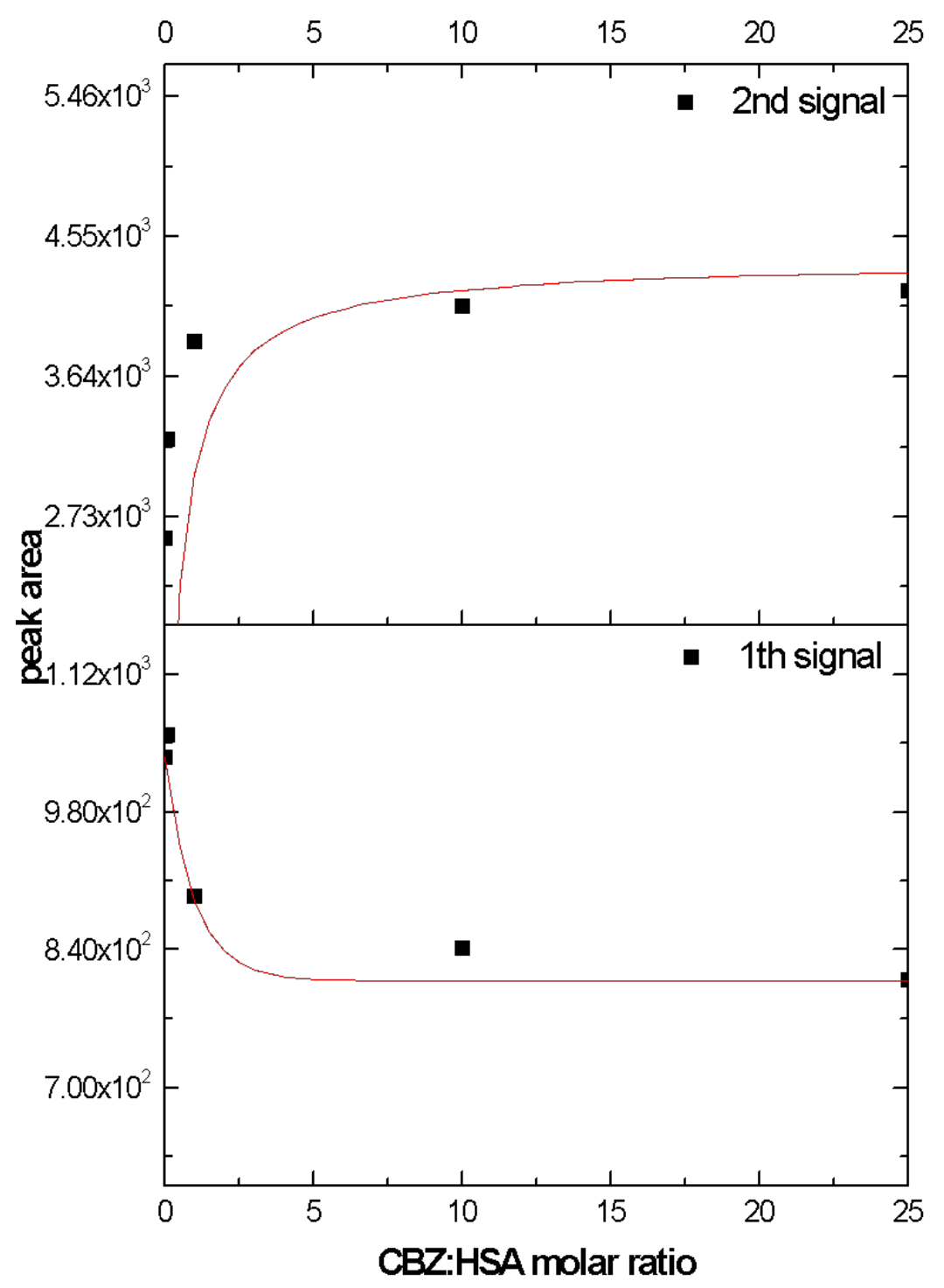

Fig. 5. Dependence between peak areas and molar ratios of a) CBZ:dHSA (pI 4.9), b) CBZ:HSA $\left(1^{\text {th }}\right.$ signal - pI $4.5,2^{\text {nd }}$ signal - pI 4.9$)$. 
a)

CBZ-dHSA

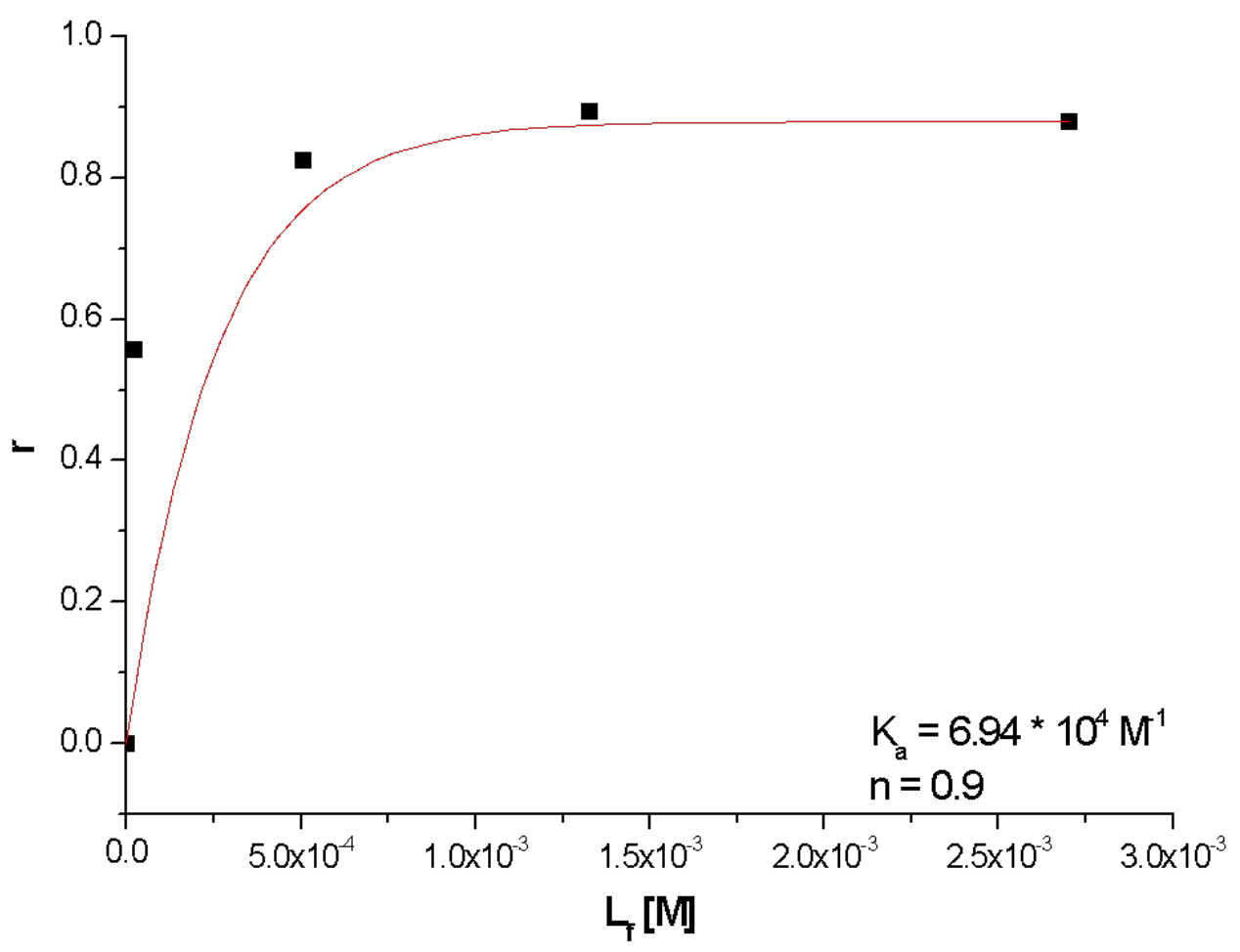

b')

CBZ-HSA

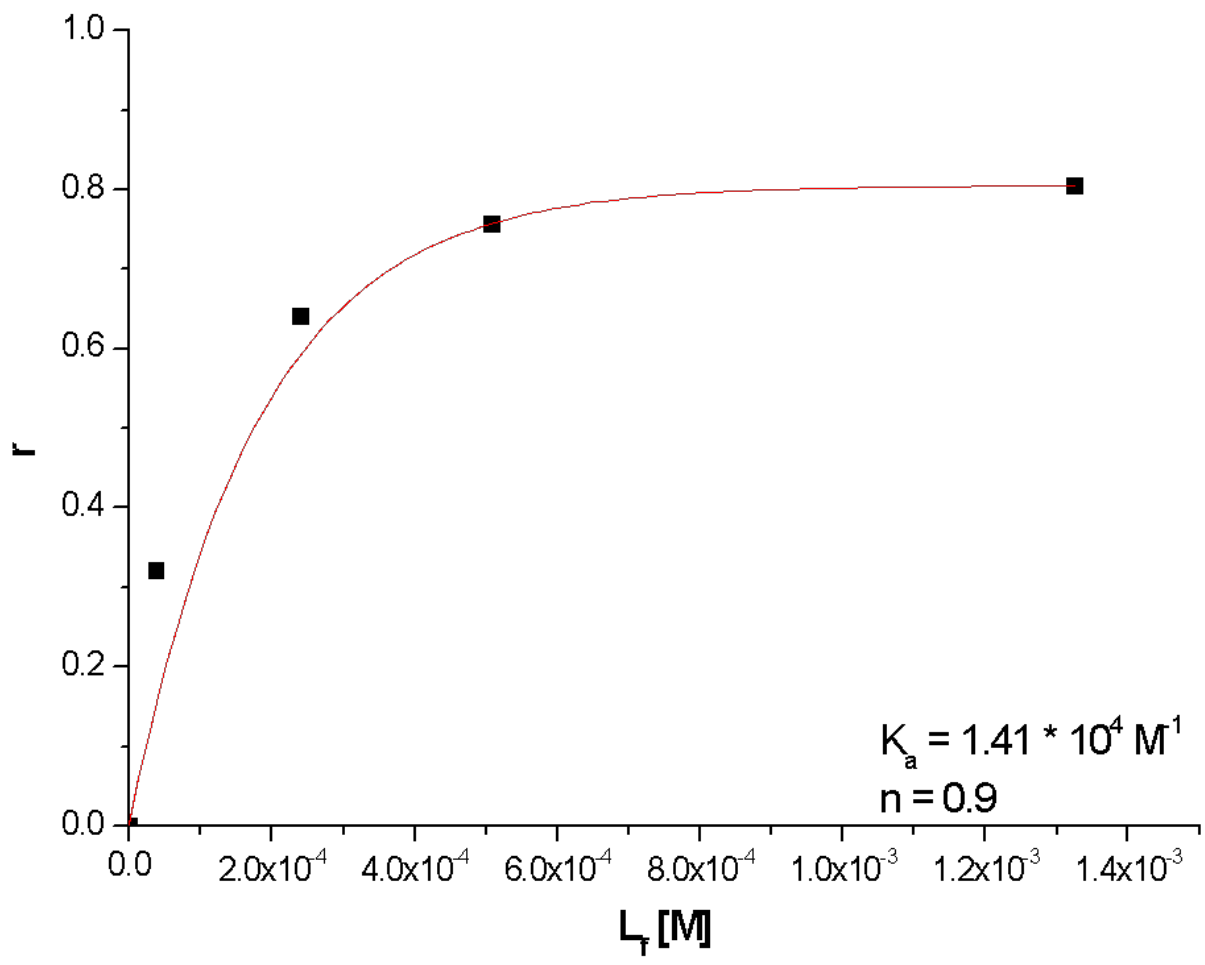


b')

CBZ-HSA

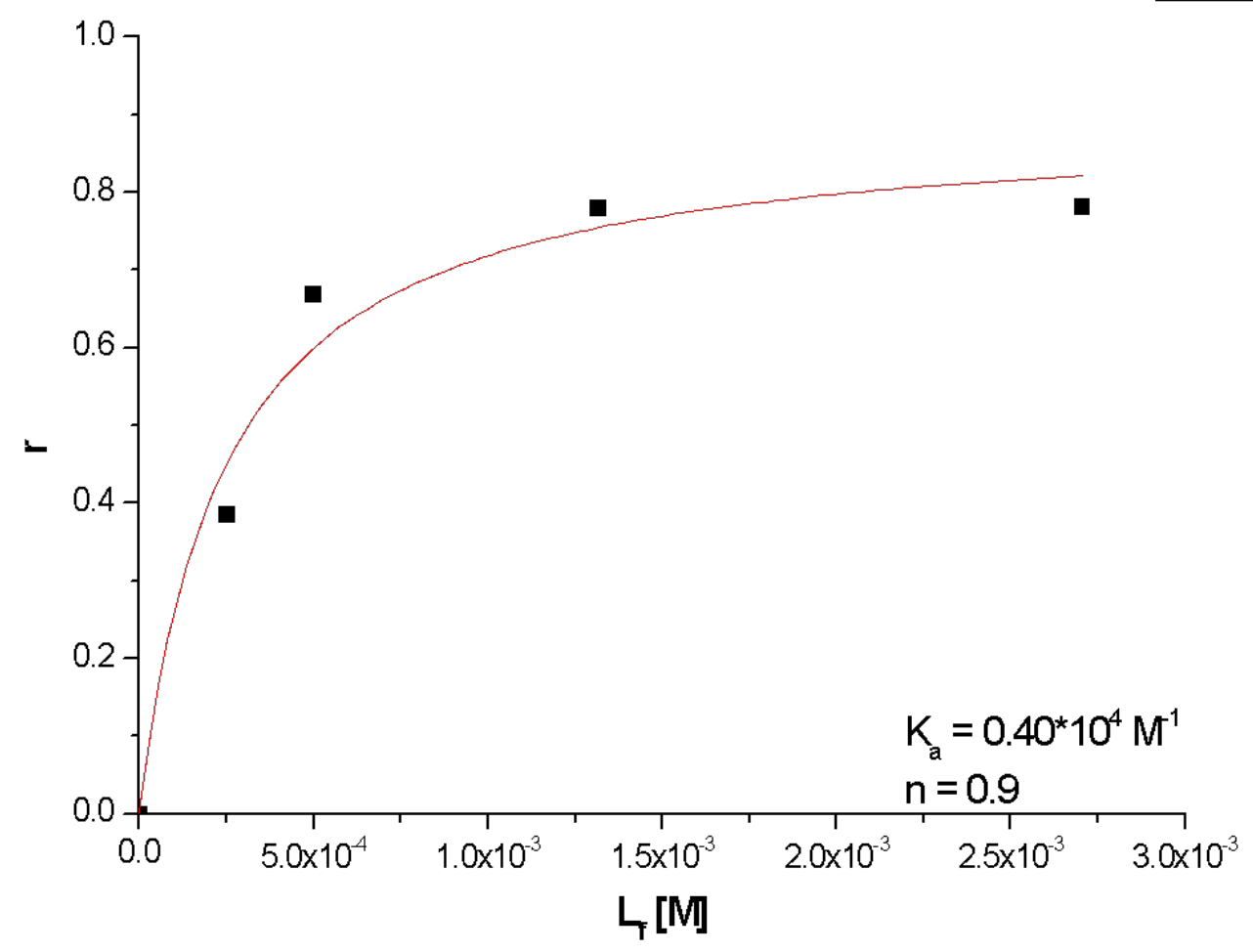

Fig. 6. Binding isotherms of a) CBZ-dHSA, b’) CBZ-HSA (pI 4.9), and b”) CBZ-HSA (pI 4.9) complexes. 